\title{
Deducing Interactions in Partially Unspecified Biological Systems
}

\author{
Paolo Baldan ${ }^{1}$, Andrea Bracciali ${ }^{2}$, Linda Brodo ${ }^{3}$, and Roberto Bruni ${ }^{2}$ \\ 1 Dipartimento di Informatica, Università Ca' Foscari di Venezia, Italia. \\ 2 Dipartimento di Informatica, Università di Pisa. \\ 3 Dipartimento di Scienze dei Linguaggi, Università di Sassari. \\ baldan@dsi.unive.it, \{braccia,bruni\}@di.unipi.it, brodo@uniss.it
}

\begin{abstract}
We show how a symbolic approach to the semantics of process algebras can be fruitfully applied to the modeling and analysis of partially unspecified biological systems, i.e., systems whose components are not fully known, cannot be described entirely, or whose functioning is not completely understood. This adds a novel deductive perspective to the use of process algebras within systems biology: the investigation of the behavioural or structural properties that unspecified components must satisfy to interact within the system. These can be computationally inferred, extending the effectiveness of the in silico experiments. The use of the approach is illustrated by means of case studies.
\end{abstract}

\section{Introduction and Motivations}

The convergence of mathematical, technical and natural science yields multidisciplinary approaches that can help in better understanding and explaining certain biological phenomena. The formal modeling of such phenomena has recently gained a lot of attention, see e.g. [31,34,20,21,17]. Some of them are based on process algebras, which provide expressive, formal descriptions, enjoy friendly syntax, compositionality and generally support software simulation. To some extent, they appear as easily accessible formalisms, particularly suited for such interdisciplinary research, that favor cross-fertilisation between the two fields: existing calculi have been sometimes applied rather directly, like in the case of stochastic semantics for the Pi-calculus [27,29], while in other cases new language primitives have been specifically designed to capture molecular and biological interaction, like the explicit treatment of membrane nesting [30], membrane activity [8], probability-based reactions [25], active sites in a protein [11], and structure-determined reactions [28,12]. These linguistic abstractions are generally complemented with suitable formal semantics that may describe system behaviour both qualitatively and quantitatively, e.g., in terms of happening reactions and their kinetic constants. 
They are generally stochastic semantics [26,32] based on Gillespie's algorithm [16]. Often, executable counterparts are provided so that system properties can be both assessed theoretically and verified by means of in silico simulations. Encouraging results, e.g. in terms of the coherence between in silico and in vitro experiments, have been obtained [22,5,10].

Within this line of research, that exploits the analogy between biological and software systems, we address the problem of dealing with qualitative analysis of open systems. In the context of computer science open systems account for components that are not fully specified or may dynamically join the system at a later stage, such as applications that access services on the network, or proprietary software components. In the biological setting unspecified components may play the part of not fully understood cellular and chemical compounds.

Here we present an application to the modeling and analysis of biological systems of symbolic transition systems [2,3], originally developed for software open systems. We aim to show that: (i) the symbolic model adds a deductive dimension to the in silico experiments, allowing one to derive the (most general) features that unknown components exhibit when interacting within a system, and (ii) the framework is language-independent, in the sense that it applies to a variety of different modeling problems at different levels of abstraction.

The main ingredients of the approach are an algebraic syntax, an operational semantics in terms of suitable labelled transitions and the possibility to deal with open and closed systems in a uniform way. The approach will be exemplified on a few case studies that exploit similar but different algebraic structures, related to different level of abstractions, granularity, and aspects of interest.

We conclude this introduction by giving an informal account of the technical notation and its usage in a small scenario comprising a virus $v$ and a cell $c$. Let $E$ be the (closed) system:

$$
E=v[\text { in c. rna }] \mid c\left[\text { open } v .\left(\text { prot } \mid r n a^{\perp}\right)\right]
$$

On the biological side, terms like $v[\ldots]$ can be understood as membraned components, while action prefixes like in $c$ models reaction capabilities. The name of the membrane identifies the kind of the component. For instance, the virus $v$ is ready to sequentially execute the actions in $c$ and $r n a$, modeling, respectively, the capability to enter a cell of kind $c$ and then to communicate some RNA information. The cell $c$ "trusts" components like $v$ and is ready to open their membranes, with action open $v$. Sometimes interaction is enabled by the exact knowledge of membrane names (i.e., the system comprises some sort of location-awareness). 
The evolution (semantics) of the system is modeled via labeled transitions from one configuration to the next. The labels record events that are visible to an external observer. The special label $\tau$ is used when the corresponding event is an internal reaction, transparent to the outside. In our example, the virus can enter the cell, the membrane $v$ is opened and the RNA interaction takes place: the compound prot $\mid r n a^{\perp}$ interacts, without further consequences in this example, with the virus RNA by means of the complementary action $r n a^{\perp}$ (while prot information is disregarded by the virus).

$$
E \rightarrow_{\tau} c\left[v[r n a] \mid \text { open } v .\left(p r o t \mid r n a^{\perp}\right)\right] \rightarrow_{\tau} c\left[r n a \mid \text { prot } \mid r n a^{\perp}\right] \rightarrow_{\tau} c[\text { prot }]
$$

The infinite set of transitions relative to all the terms of the calculus can be finitely specified by a set of structured operational semantics (sos) rules For instance, all the transitions about a membraned component $m[$ in $n . Q \mid R]$ entering the membrane $n[P]$, or a component open $n . Q$ destroying the membrane of $n[P]$ are respectively modelled by rules (in) and (open) above, valid for all $m, n, P, Q, R$. Analogously, if any two components $P_{1}$ and $P_{2}$ can exhibit complementary actions $\alpha$ and $\alpha^{\perp}$, then by rule (comm) their reaction generates a $\tau$ transition.

Now imagine that the content of the virus cannot be fully characterised, e.g., because it has not been fully understood. In this case we regard $E$ as an environment: an open biological system modeled as a term with place-holders $X$, whose unknown components can only be disclosed dynamically (e.g. when they react to or provoke certain stimuli) or where components (i.e., closed systems) or other sub-environments can be dynamically plugged in.

$$
E[X]=v[X] \mid c\left[\text { open } v \cdot\left(\text { prot } \mid r n a^{\perp}\right)\right]
$$

One possibility is to study all the closures $E[p]$ of $E[X]$ w.r.t. all the possible closed component $p$. When simulation is attempted in silico, then infinitely many $p$ must be considered. Moreover, conceptually, this approach prevents the dynamics disclosure of environments to be considered, since they are fully disclosed at the beginning.

Symbolic transition systems (sTs) allow environments as states and logic formulae as transition labels. They exploit the idea that the behaviour of $E[X]$ depends on the applicable semantic rules, which can be partly determined by means of the known structure of $E[X]$ itself, and may, in turn, impose a requirement over $X$ in order to apply the rule. The formulae of sTs transitions, which annotate unknown components with their relevant 
behavioural or structural requirements, can be composed throughout an execution trace of the environment and represent the "inferred" constraints that an unknown component must fulfill to drive the system to a given state, e.g. to attack problems like predicting the environmental conditions that let a virus reproduce. For instance, the open system $E[X]$ can evolve via suitable "abstractions" of the transitions in (2) for the closed $E$ :

$E[X] \stackrel{\text { inc.YYZ }}{\longrightarrow} c\left[v[Y \mid Z] \mid\right.$ open v.(prot $\left.\left.\mid r n a^{\perp}\right)\right] \stackrel{Y, Z}{\longrightarrow}_{\tau} c\left[Y|Z|\right.$ prot $\left.\mid r n a^{\perp}\right] \stackrel{\diamond r n a W, Z}{\longrightarrow} c[W|Z|$ prot $]$

The first one exhibits the formula in c.Y $\mid Z$ : the unspecified component $X$ should "at least" be able to perform in c (hence $X$ must "know" c) and then behave as $Y \mid Z$, as required by rule (in). The second one imposes no constraints since the environment evolves autonomously, the third one requires on $Z$ the capability to interact by means of $r n a$.

Note that the formula in c.( $\diamond r n a . W \mid Z)$, obtained by composing the formulae along the execution trace, recaps in a general format the capabilities required to $X$ in order to carry on the overall interaction within the environment (and it is satisfied by the component $p=$ in c.rna $\equiv$ in c. $($ rna.0 $\mid 0)$ that instantiates $E[X]$ to $E[p]=E)$. Formulae composition is especially relevant for the analysis of the evolution traces of partially specified bio-environment.

Synopsis. In $\S 2$ we recall the basics of sts. The framework for the analysis of bio-processes is illustrated in $\S 3$ by discussing, in two examples, how it can be used to reason with incomplete information. The first example is based on an original (simplified) formalisation of the life cycle of the $\lambda$-phage virus in BioAmbients. The second example deals with a model of viral cell infection, originally from [1] and used in [8] to introduce Brane Calculi. Concluding remarks and future perspectives are in $\S 4$. The presentation is mainly of informal nature. For the convenience of the reader, some formal details and complementary notions have been inserted in an Appendix.

\section{Symbolic Operational Semantics}

This section recalls the key definitions about symbolic transition systems. A more comprehensive formal presentation can be found in $[2,3]$. In what follows, $E[X]$ abbreviates $E\left[X_{1}, \ldots, X_{n}\right]$, a generic environment with $n$ variables. The sketched virus example will exemplify formal definitions.

Definition 1 (Symbolic Transition System). A symbolic transition system (sTs) $\mathcal{S}$ is a set of transitions 


$$
C\left[X_{1}, \ldots, X_{n}\right] \stackrel{\left(\varphi_{1}, \ldots, \varphi_{n}\right)}{\longrightarrow}{ }_{a} D\left[Y_{1}, \ldots, Y_{m}\right]
$$

where $C[X]$ and $D[Y]$ are environments, a an action label and $\varphi_{i}$ are formulae over variables $\left\{Y_{1}, \ldots, Y_{m}\right\}$ (in a suitable logic, as defined below).

Informally, a symbolic transition represents the fact that the environment $C[X]$ can exhibit an action $a$ and evolve to $D[Y]$ whenever the holes $X$ are filled with any components satisfying $\varphi$. The label $\varphi$ should encode the "least necessary" conditions that components should fulfill for properly taking part to the transition. For the sake of this presentation, following [2], we exploit the logic SL, with action and structural modalities in the style of the ambient logic [9]. However, different choices are conceivable, depending on the calculus of interest.

Spatial modalities emerge when, in order to perform a transition, an environment $E[X]$ must match the left-hand side of the conclusion of a rule. This may require a certain structure to the components that may possibly be plugged in, hence requiring the constructors of the calculus, like - I _ or $n$ [_], to appear as terms of the logic, which we call spatial operators. Furthermore, the premises of the matched rule must be satisfiable. Such premises may typically require each plugged component to be able to exhibit some behaviour, as in rule (comm). Hence, the logic also includes modal operators $\diamond a_{\text {_ }}$ expressing the capability to perform an action $a$. A formula which does not impose any constraint on the component is represented as a logical variable $X$, called the residual placeholder.

Definition 2 (SL). The formulae of the sTs logic SL are

$$
\varphi::=X|\diamond a \varphi| f\left(\varphi_{1}, \ldots, \varphi_{n}\right)
$$

where $X$ is a residual pleaceholder, $a$ is an action and $f$ is a spatial operator. A component $p$ satisfies the formula $\varphi$, if $p \vDash \varphi$ holds according to the rules:

$$
\begin{array}{ll}
p \vDash X & \\
p \vDash f\left(\varphi_{1}, \ldots, \varphi_{n}\right) & \text { if } \exists p_{1}, \ldots, p_{n} \cdot p \equiv f\left(p_{1}, \ldots, p_{n}\right) \wedge \forall i . p_{i} \vDash \varphi_{i} \\
p \vDash \diamond a \varphi & \text { if } \exists q . p \rightarrow a q \wedge q \vDash \varphi
\end{array}
$$

For example, the component $p=a^{\perp} .0 \mid a . b .0$ satisfies the formula $\diamond a X$, written $p \vDash \diamond a X$, because $p \rightarrow_{a} a^{\perp} .0 \mid b .0$ and $a^{\perp} .0 \mid b .0 \vDash X$.

Given a formula $\varphi$ and $n$ components $q_{1}, \ldots, q_{n}$, we denote by $\varphi\left[q_{1} / X_{1}, \ldots, q_{n} / X_{n}\right]$, the formula obtained from $\varphi$ by replacing variables $X_{i}$ with components $q_{i}$. Analogously, we denote by $\varphi\left[\varphi_{1} / X_{1}, \ldots, \varphi_{n} / X_{n}\right]$ the formula obtained from $\varphi$ by replacing variables $X_{i}$ with formulae $\varphi_{i}$. Variables in formulae can stand for the residual $q$ of $p$, after that $p$ has exhibited the capabilities and/or the structure imposed by the formula. 
Definition 3 (Satisfaction with residuals). Let $p$ be a component, $q=$ $q_{1}, \ldots, q_{n}$ a tuple of components and $\varphi \in \mathrm{SL}$ a formula whose variables are contained in $\left\{X_{1}, \ldots, X_{n}\right\}$. Then, $p$ satisfies $\varphi$ with residuals $q_{1}, \ldots, q_{n}$, written $p \vDash \varphi ; \boldsymbol{q}$, whenever $p \vDash \varphi\left[q_{1} / X_{1}, \ldots, q_{n} / X_{n}\right]$.

For example, if $p=n\left[a^{\perp} .0 \mid a . b .0\right]$ and $\varphi=n\left[\diamond a^{\perp} X_{1} \mid a . X_{2}\right]$, then trivially $p \vDash \varphi$, and if $q_{1}=0$ and $q_{2}=b .0$ then $p \vDash \varphi ;\left(q_{1}, q_{2}\right)$. We shall write $\boldsymbol{p} \vDash \varphi$; $\boldsymbol{q}$ where $\varphi=\varphi_{1}, \ldots, \varphi_{k}$ and $\boldsymbol{p}=p_{1}, \ldots, p_{k}$ are tuples of formulae and components, respectively, with the obvious meaning that $p_{i} \vDash \varphi_{i}\left[q_{1} / X_{1}, \ldots, q_{n} / X_{n}\right]$ for all $i \in\{1, \ldots, k\}$.

The proper correspondence between the transitions of environments and those of components is established by suitable soundness and completeness properties, for which we refer the interested reader to [2,3]. Notably, some sort of "standard" sound and complete sTs can be derived for a large class of process calculi, whose operational behaviour is defined by a set of sos rules in a suitable format [3]. Such sTs can be constructed by means of a unification-based process regarding the states of the STS and the rules. We remark that all symbolic transitions spawn from $E[X]$ in (3) are sound.

\section{Reasoning with Incomplete Information}

We apply now the sTss framework to two case studies (their LTS semantics are reported in the Appendix). The first one is an original formalization of a pattern of protein interaction relative to the $\lambda$-phago virus. Starting from an incomplete BioAmbients specification of the system, the behaviour of one of the proteins can be further characterised by reasoning symbolically on the dynamics of the system. The second example, split in two parts, consists of the symbolic reading of a paradigmatic example of biological interaction, also used to introduce the Brane Calculi in [8]. Here, pretending cell reactions to viruses are not fully understood, we infer the same behaviour described in the original example. Moreover, without changing the experiment, we additionally deduce the (known) mechanisms allowing a given protein to block the virus. Along the line of process algebras, these examples also demonstrate the applicability of our approach to different levels of abstraction, and its corresponding versatility in supporting the right representation language according to the problem at hand (although they have required a bit of notation). 

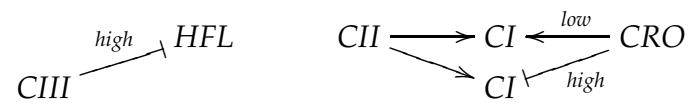

Fig. 1. Hypothesis on inhibition and activation roles of $C R O$ and $C I I I$ proteins.

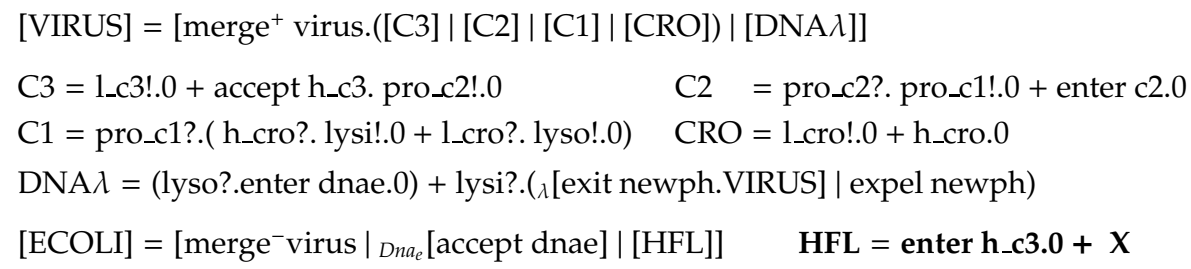

Fig. 2. Partial specification of [VIRUS] and [ECOLI]

\subsection{Protein Interaction: $\lambda$-phage life-cycle}

$\lambda$-phage simplified life cycle. We consider a simplified representation of the $\lambda$-phage virus. This virus replicates by binding with the E.coli bacterium and injecting its DNA into the bacterium cell. Then, either the virus replicates in several copies until the bacterium membrane is destroyed and the copies released (lytic pathway), or the virus DNA merges into the bacterium DNA, the infected bacterium cell multiplies, and its offspring may themselves eventually end up in a lytic pathway (lysogenic pathway). The selection of one or other pathway is determined by the interaction of the CRO, CI, CII, CIII and HFL proteins in the bacterium cell.

We study the system assuming the following knowledge (see Fig. 1). A high concentration of $\mathrm{CI}$ determines the lysogenic cycle, its absence the lytic one. The production of CI is promoted by CII, if not inhibited. The role of the bacterial protein HFL is not fully understood, although we know that it can be inhibited by a high concentration of CIII. Moreover, a low concentration of $\mathrm{CRO}$ directly stimulates the production of $\mathrm{CI}$, while a high concentration of it destroys $\mathrm{CI}$. Hence, the lysogenic cycle can be characterised as low $\mathrm{CRO}$ and high $\mathrm{CIII}$ concentrations (first row of the figure), while the lytic one seems to depend on high CRO, exclusively (second row).

BioAmbients uncomplete specification. Under the above hypotheses, the virus and the bacterium can be naturally represented in the BioAmbient calculus as two membraned systems, as shown in Fig. 2 (a formal description of the BioAmbient calculus can be found in App. A). Proteins are represented as membranes (written [...]) "delimiting" the behaviour they can express. They interact at the same level of nesting: activation is modeled as communication (input/output 
Ecoli $\left[([\mathrm{C} 3]|[C 2]|[C 1] \mid[C R O])|[D N A \lambda]|_{D_{n n a_{e}}}[\right.$ accept dnae $] \mid$ [enterh_c3.0 + X]]

$\stackrel{\left(I\left\llcorner c 3 ? Y_{1}+Y_{2}\right) \mid Y_{3}\right.}{\longrightarrow}$ Ecoli $[\mathrm{CIII}[0]|[\mathbf{C 2}]|[C 1] \mid[C R O])|[D N A \lambda]|{ }_{\text {Dnae }}[$ accept dnae $\left.] \mid \mathbf{h f l}_{\mathbf{h f l}}\left[\mathbf{Y}_{\mathbf{1}} \mid \mathbf{Y}_{3}\right]\right]$

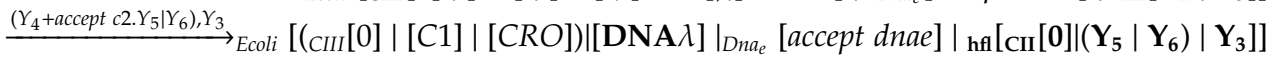

$\stackrel{\left(Y_{7}+l y s i ! Y_{8}\right), Y_{6}, Y_{3}}{\longrightarrow}{ }_{E c o l i}\left[(C I I I[0]|[C 1]|[C R O]) \mid\left[{ }_{\lambda}[\right.\right.$ exit newph.VIRUS $] \mid$ expel newph] $\mid$

$\left.{ }_{\text {Dnae }}[$ accept dnae $\left.]\right|_{h f l}\left[\mathrm{CII}[0]\left|\left(Y_{8} \mid Y_{6}\right)\right| Y_{3}\right]\right]$

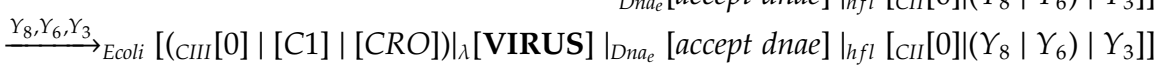

pairs of actions [..pro_c2?..]|[..pro_c2!..]) and inhibition as encapsulation $\left(\left.{ }_{1}[.\right.$. enter $a .]\right|_{2}.[.$. accept $a .$.$\left.] that evolves in { }_{2}[. .1[.] .].\right)$, since this technically blocks the capability of the enclosed protein to communicate in its original environment. The virus consists of the capability to penetrate a suitable membraned environment ([merge ${ }^{+}$virus..]), i.e., the bacterium cell ([merge $e^{-}$virus..]), and then expose its DNA and express proteins. CIII can either signal a low concentration or enclose HFL (or any compatible protein) then activating CII by means of a suitable communication. Once activated, CII promotes CI. Moreover, the possibility of CII being itself inhibited has also been modeled (enter c2). Sensitivity to high or low concentrations of $\mathrm{CRO}$, modeled by means of suitable communications, causes CI to emit either the lysogenic or the lytic activation signal. This is received by the virus DNA which, accordingly, either enters the bacterium DNA, or expels into the bacterium cell a copy of the virus. The bacterium is modeled as a membrane that can be injected by a virus and contains membraned DNA, which can be accessed by other suitable DNA, and the HFL protein. Importantly, this is represented as a partially specified component, which, as we know, can be inhibited by CIII (enter $h_{-} c 3$ ) but also could alternatively exhibit a behaviour we are not able to specify at the present, represented as variable $X$.

Symbolic transition system. We study the possible evolutions of the un-completely specified system ${ }_{\lambda}[$ VIRUS $] \mid$ Ecoli[ECOLI] (bio-ambients are sometimes labeled for clarity) in order to understand the possible interactions of HFL within it. In the corresponding sTs we can find the trace above. (As mentioned in $\S 2$, the labels can be automatically constructed on the basis of the BioAmbient proof rules, while the logic simply consists of the modal operator $\diamond_{\rightarrow}$, which stands for the possibility of performing an unlabelled transition, and of the spatial operators deriving from the syntax of the calculus). The composition of the formulae over the trace yelds an interesting characterisation of the behaviour of HFL:

$$
\left(l_{-} c 3 ? .\left(Y_{4}+\text { accept } c 2\left(Y_{7}+\text { lysi!. } Y_{8}\right) \mid Y_{6}\right)+Y_{2}\right) \mid Y_{3}
$$

It is possible to see that this is a correct abstraction of the actually known HFL (reported in (10) App. A.1), and the above symbolic trace is an abstraction of the corresponding ground trace ((12)-(16) App. A.1). 
Moreover, this allows us to complete the picture of protein interactions of Fig. 1 with the relation between low CIII, HFL and CII (the interaction schema is updated in Fig. 4), although the partial specification adopted did not have any information about this specific point.

\subsection{Cellular Interaction: Membrane Trepassing}

We model an abstraction of a virus replicating its RNA by exploiting a host cell (this has been more exhaustively treated in [8]). The virus membrane complex contains the capsid another membrane complex, which encloses the nucleocapsid, i.e. the cytoplasme containing the viral RNA. Here we model the endocytic pathway: the virus penetrates the cell membrane.

We assume that the behaviour of the virus membrane is not known and deduce it from the operational rules describing the behaviour of the cell. We adopt $\mathrm{fBC}$, a simplified version of the Brane Calculi, which more suitably models this example. Indeed, $\mathrm{fBC}$ focuses on membrane interactions, within the scope of this section, and molecular interactions in the next one. Brane Calculi are intended to model biological interactions inspired by endocytosis/exocytosis, indicated in [8] as bitonal interactions, since, informally speaking, they preserve a periodicity between inner and outer areas of membranes.

The calculus fBC (see App. B for details) can be understood as an extension of BioAmbients, where membranes exhibit themselves a behaviour. The basic membrane complex $\sigma[P]$ consists of an active external membrane layer $\sigma$ and of complex $P$ inside the membrane $(\diamond$ is the null membrane complex). Other complexes can be obtained by the composition of $P$ and $Q$, written $P \circ Q$, or as a multiset of molecules, $m_{1} \circ \cdots \circ m_{k}$. Interaction between membrane complexes happens through the active membrane layer $\sigma$, which can be halted 0 , an action prefixed to an active layer $a . \sigma$ and the parallel composition of active layers $\sigma \mid \tau$. Membranes behave as follows. $\sigma[P]$ can enter $\tau[Q]$, if $\sigma$ can execute a $p_{n}$ action and $\tau$ the corresponding coaction $p_{n}^{\perp}(\rho)$ ( $n$ is the same) and $\sigma[P]$ is enclosed within the active membrane $\rho$, according to the spirit of bitonal reactions (phago). In $\tau[\sigma[P] \circ Q]$ the subsystem $P$ can leave the $\tau[\ldots]$ membrane complex if $\sigma$ and $\tau$ are ready to execute, respectively, $e_{n}$ and $e_{n}^{\perp}$ (exo). Finally, $\sigma[P]$ and $\tau[Q]$ merge in $\sigma \mid \tau[P \circ Q]$ if the membranes can execute $m_{n}$ and $m_{n}^{\perp}$ respectively (mate).

fBC formalisation. Via a phagocytosis the virus enters the cell wrapped by a membrane. Then, the external membrane of the virus merges with 
$\varphi::=X|\psi[\varphi]| \varphi \circ \varphi\left|\diamond_{-} \varphi\right| \theta$ (complexes)

$\alpha::=p_{n}\left|p_{n}^{\perp}(\psi)\right| e_{n} \mid e_{n}^{\perp} \quad$ (actions)
$\psi::=Y|\psi| \psi \mid \alpha . \psi$ (membranes) $\theta::=Z|m| \theta \mid \theta$ (molecules)

a component of the cell, the endosome. Finally, through an exocytosis, the viral nucleocapsid, and the viral RNA it contains, is released directly in the cytosol of the cell. Let us suppose that the mechanisms in the virus membrane are not very well understood. We represent this with the following partial specification, having variable $Y$ in place of the virus membrane.

$$
\begin{array}{rrr}
\text { virus }=\mathbf{Y}[\text { nucap }] & \text { nucap }=\text { capsid }[\text { vRNA }] & \text { capsid }=p_{b} \mid \text { bud } \mid \text { disasm } \\
\text { cell }=p_{a}^{\perp}\left(m_{a}\right) \mid e_{b}^{\perp}[\text { cytosol }] & \text { cytosol }=\text { endosome } \circ \text { CC } & \text { endosome }=m_{a}^{\perp} \mid e_{a}^{\perp}[\odot]
\end{array}
$$

The virus content nucap is known and it will take part to later stages. It consists of a membrane complex, which contains the RNA and whose active part, capsid, is ready to execute a phago action $p_{b}$, a disasm set of actions that will be defined later, and a bud action that is not relevant here. The cell membrane is ready for a phago $p_{a}^{\perp}\left(m_{a}\right)$ and an exo $e_{b}^{\perp}$ action (for the reproduced virus eventually leaving the cell). Its content, cytosol, consists of a part, denoted CC, here not relevant, and the endosome, i.e. a membrane complex that can merge $m_{a}^{\perp}$ with what has been phago-ed and it can uncoat its content $e_{a}^{\perp}$, in case a suitable coaction can be provided, possibly by the virus.

Symbolic transition system. Also in this example, the associated logic is straightforwardly induced by the syntax of $\mathrm{fBC}$, as shown above. Note that, being $\mathrm{fBC}$ semantics unlabelled, the modality $\diamond_{-} \psi$ simply stands for the capability of executing any action (e.g., as it may be required by the (mem) rule, see App. B). Then, we study the environment:

$$
F[Y]=Y[\text { nucap }] \circ p_{a}^{\perp}\left(m_{a}\right) \mid e_{b}^{\perp}[\text { cytosol }]
$$

where $Y$ stands for the unknown virus membrane. A possible symbolic trace of the sTs of $F[X]$ is:

$$
\begin{array}{r}
\left.F[Y] \stackrel{p_{a} \cdot Y_{1} \mid Y_{2}}{\longrightarrow} e_{b}^{\perp}\left[m_{a}\left[Y_{1} \mid Y_{2}[\text { nucap }]\right] \circ m_{a}^{\perp} \mid e_{a}^{\perp}[\odot] \circ C C\right]\right] \\
\stackrel{Y_{1}, Y_{2}}{\longrightarrow} e_{b}^{\perp}\left[e_{a}^{\perp}\left[Y_{1} \mid Y_{2}[\text { nucap }]\right] \circ C C\right] \\
\stackrel{e_{a} . Y_{3} \mid Y_{4}, Y_{2}}{\longrightarrow} e_{b}^{\perp}\left[Y_{3}\left|Y_{4}\right| Y_{2}[\odot] \circ \text { nucap } \circ C C\right]
\end{array}
$$

The first symbolic transition (5) constrains the virus membrane to be able to perform a phago $p_{a}$ action in order to enter the cell via endocytosis, $Y=p_{a} . Y_{1} \mid Y_{2}$. The requirement is specific for the action offered by the cell membrane. The second symbolic transition (6) does not involve the virus membrane, since the nucap of the virus can merge with the cytosol of the cell without imposing any further condition on the viral membrane. 
The formula hence reverts to identity $Y_{1}, Y_{2}$ : no requirements over the unspecified components, since the rest of the system is able to evolve autonomously. The last transition (7) requires $e_{a} . Y_{3} \mid Y_{4}, Y_{2}$, i.e. the (current state of the) virus membrane should be able to exhibit an action $e_{a}$ in order to uncoat its content nucap by means of exocytosis. The constraint $Y_{1}=e_{a} \cdot Y_{3} \mid Y_{4}$ is the most general, coherently with the semantic rules.

Finally, inferred information about the unknown components, when they contribute to the overall system behaviour, can be gathered by composing the logical formulae used as labels: any virus whose membrane satisfies $\psi_{\mathrm{sTs}}=p_{a} .\left(e_{a} \cdot Y_{3} \mid Y_{4}\right) \mid Y_{2}$ will be able to enter in the cell and release its nucap. Note that $\psi_{\text {sтs }}$ characterises a general class of components which allow for the interaction of interest. For instance, not only the membrane of the virus $p_{a} \cdot e_{a}\left[\right.$ nucap ] but also the membranes $p_{a} \cdot\left(e_{a} \mid p_{a} \cdot e_{a}\right)$ or $m_{n} \mid p_{a} \cdot e_{a}$ satisfy $\psi_{\text {sts }}$ and, once plugged in $Y$, are sufficient to drive the system through the same kind of behaviour (and maybe others).

\subsection{Biochemical Interaction: Viral RNA Replication}

Once inside the cell, the virus capsid is removed (uncoating process), and the virus RNA replicates. Besides this process, also discussed in [8], we address virus neutralisation by the cell. Here, we assume that the mechanisms in the cell content are not fully understood and we show how information about the cell content, relevant for virus replication or neutralisation, can be inferred.

The $\mathrm{fBC}$ calculus is extended in order to express a level of abstraction adequate for the biochemical level: a bind and release action, denoted $r_{1}\left(r_{2}\right) \rightrightarrows s_{1}\left(s_{2}\right)$, is added to let membranes interact with molecules. The action can be executed by a membrane if the molecules $r_{2}$ are contained in the membrane complex and the molecules $r_{1}$ are present outside it. Its effect is to substitute the molecules $r_{1}$ with the molecules $s_{1}$ outside, and $r_{2}$ with the $s_{2}$ inside (see the rule in App. B)

The logical formulae for actions are correspondingly extended as $\alpha::=p_{n}\left|p_{n}^{\perp}(\psi)\right| e_{n}\left|e_{n}^{\perp}\right| \theta(\theta) \rightrightarrows \theta(\theta)$.

We study again a partially specified cell, and then we show how the inferred constraints are coherent with actual components that can reasonably play the part of the unspecified ones.

Virus replication. We assume that the virus $p_{a} \cdot e_{a}$ [ nucap ], which fulfills the characterisation inferred in $\S 3.2$, has entered the cell, reaching the state $e_{b}^{\perp}\left[p_{b}|b u d|\right.$ disasm [vRNA] $\left.\circ C C\right]$ (a coherent instance of the one in (7)). The action disasm, responsible of uncoating the $v R N A$, is specified 
as a $b \& r$ action activated by the presence of an outer trigger. It moves the inner $v R N A$ outside: $\operatorname{disasm}=\operatorname{dis} \operatorname{Tr} g(v R N A) \rightrightarrows v R N A(\diamond)$. Moreover, we suppose that the remaining cell content $C C$ is not fully understood, i.e. we focus on the environment

$$
G[X]=e_{b}^{\perp}\left[p_{b}|b u d| \operatorname{disTrg}(v R N A) \rightrightarrows v R N A(\diamond)[v R N A] \circ X\right]
$$

A possible symbolic trace of $G[X]$ is the following:

$G[X] \stackrel{\text { disTrgo }}{\longrightarrow} X_{0} e_{b}^{\perp}\left[p_{b} \mid b u d[\diamond] \circ v R N A \circ X_{0}\right] \stackrel{\xi}{\longrightarrow} e_{b}^{\perp}\left[p_{b}\left|b u d[\diamond] \circ Z_{2} \circ Y_{4}\right| Y_{5}\left[Z_{3} \circ X_{6}\right] \circ X_{7}\right]$

The applicable $(b \& r)$ rule justifies the first symbolic transition with a spatial constraint requiring that $X$ contains at least a disTrg molecule in order to trigger the removal of the viral capsid. The second symbolic transition is justified by the $v R N A$ molecule, now free within the cell, used as a trigger for another application of $(b \& r)$, where $\xi=v R N A\left(Z_{1}\right) \rightrightarrows Z_{2}\left(Z_{3}\right) . Y_{4} \mid Y_{5}\left[Z_{1} \circ X_{6}\right] \circ X_{7}$. The formula $\xi$ implies that, triggered by the outer presence of $v R N A$, a set of molecules $Z_{2}$ can be released within the cell, so that $v R N A$ replication can be supported by the cell. The composition of the formulae in (8) yields

$$
\Psi_{\text {srs }}=\operatorname{disTrg} \circ v R N A\left(Z_{1}\right) \rightrightarrows Z_{2}\left(Z_{3}\right) . Y_{4} \mid Y_{5}\left[Z_{1} \circ X_{6}\right] \circ X_{7}
$$

As expected, $\Psi_{\text {STS }}$ characterises the mechanisms of virus replication as modeled in [8], which we are following. There, $C C$ is read as providing the suitable triggering and replication (two $v R N A$ released) capability:

$$
C C=\operatorname{disTrg} \circ v R N A r e p l \circ C C^{\prime} \quad v R N A r e p l=v R N A(\diamond) \rightrightarrows v R N A \circ v R N A(\diamond)[\diamond]
$$

Importantly, the above definition satisfies the characterisation $\Psi_{\text {STS }}$ obtained by reasoning symbolically, i.e., $C C \vDash \Psi_{\text {STS }}$ (where $Z_{2}$ stands for $v R N A \circ v R N A, Z_{1}, Z_{3}, X_{6}, X_{7}$ for $\diamond$, and $Y_{4}, Y_{6}$ for 0$)$. Moreover, the behaviour of $G[C C]$ comprises a trace that is an instance of the symbolic (8), leading, as expected, to a state where $v R N A$ has been replicated: $e_{b}^{\perp}\left[p_{b} \mid b u d[\odot] \circ v R N A \circ v R N A \circ C C^{\prime}\right]$.

Virus neutralisation. It is interesting to observe another possible evolution of $G[X]$, justified in the corresponding sTs by a (phago) rule

$$
G[X] \stackrel{\psi}{\longrightarrow} e_{b}^{\perp}\left[Y_{2} \mid Y_{3}\left[Y_{1}[\text { bud } \mid \operatorname{disasm}[v R N A]] \circ X_{4}\right]\right]
$$

with $\psi=p_{b}^{\perp}\left(Y_{1}\right) \cdot Y_{2} \mid Y_{3}\left[X_{4}\right]$. In this case the $p_{b}$ action of the virus membrane has been exploited to trap the virus nucap within a membraned complex $\left(Y_{1}[]\right)$.

This evolution, not considered in [8], mimics the presence of a $M x$ like protein in the cell that inhibits the replication of the virus. This type of proteins seems to play an antiviral activity by trapping the viral capsid and moving it in a location of the cell where the mechanism 
for the generation of new virus particles becomes unavailable [18]. The simplest representation of the $M x$ protein can be drawn from $\psi$ as $M x=$ $p_{b}^{\perp}(0)[\odot]$ : by means of $p_{b}^{\perp}(0)$ the viral nucap is trapped within an empty membrane. $M x$ seems to be a coherent simplification of the actual known behaviour of the protein, while $\psi=p \frac{\perp}{\perp}\left(Y_{1}\right) . Y_{2} \mid Y_{3}\left[X_{4}\right]$ characterises the cell components (including the protein $M x$, but possibly others) capable of trapping the virus within a membrane. Adding the $M x$ protein to a cell with the RNA replication mechanism determine an LTS reaching a state where the virus has been phago-ed in a membrane where it can not reproduce (the $M x$ membrane has been annotated for readability): $e_{b}^{\perp}[0 \overline{0} \overline{0}[$ bud $\mid \operatorname{disasm}[v R N A]] \overline{]} \circ R C]$.

While the behaviour of $M x$ proteins has been studied elsewhere, it is worth noting that here it has been inferred from the general rules defining the calculus and an incomplete initial specification. The same specification has led to the inference of the virus replication mechanism. This experiment shows how symbolically reasoning appears as a deductive mechanism, suitable to infer unknown information.

\subsection{Discussion}

We have presented two proof of concept examples of the application of sTss to biological problems. The former is an original formulation of biological interaction that highlights the problem of understanding the interplay of a protein network. The latter is a paradigmatic example of the interaction between a virus and its host cell. These examples have illustrated how, reasoning in presence of incomplete specifications, it has been possible to deduce new knowledge about the studied systems, like - the emergence of unspecified interactions between bio-components, e.g. the possibility of a HFL-like protein to determine the lytic or lysogenic cycle in the $\lambda$-phage virus life-cycle;

- the constraints over the behaviour and the structure of bio-components needed to participate to the evolution of a system, e.g. the need of the cell content to provide a trigger for $v R N A$ replication;

- the discovery of possible components or behaviour not explicitly foreseen in the initial specification of the system under analysis, e.g. the existence and behaviour of an $M x$-like protein blocking virus replication.

This poses the problem of characterising the (reachability of) relevant states and evolution traces of the partially specified bio-environments, and, analogously, of proving bio-system properties. For instance one might want to exploit the synthesized sTsfor the automated state-space 
exploration in order to characterise "unknown" dangerous bioagents that can compromise the regular activity of a cellular system. An interesting approach in this sense is the definition of a modal logic and a model checking algorithm for Brane Calculi [24] (along the line of Ambient Logic [9]), which defines spatial and temporal properties on membrane systems. Similarly, $[13,14,35]$ exploit Pathway logic and matching algorithms for model checking the evolutions of biological systems. However, beyond similarities, e.g. formulas as labels and unification/matching algorithms, our theoretical framework poses the problem of model checking open-ended systems. This is an interesting problem under investigation, whose scope is beyond this paper.

Formulae relative to traces play the part of (minimal) necessary conditions that unspecified components must fulfill to possibly drive the system through the trace. Trivially, if $p \vDash \phi$ and $q \vDash \psi$ then $p+q$ satisfies both of them and can lead the system through possibly completely unrelated evolutions. This suggests, in general, the difficulties in associating processes to desired behaviours. Besides, while $p+q$ is definitely a process in abstract terms, it might not be feasible in biological terms, so that process characterisation could also require domain specific solutions. This issue is under investigation.

\section{Concluding Remarks and Future Work}

We have proposed the application of a symbolic semantics for process calculi to the modeling of open biological processes, with the aim of enabling the reasoning about biological system specifications where some components or features are unknown. This lies within a multidisciplinary line of research across software and biological open systems.

An open biological system is seen as a partially specified process of a given calculus suited for modeling biological processes. Its semantics is given in terms of symbolic transition systems (STS), whose transitions are labeled with logical formulae that express the structural and/or behavioural requirements over the unknown components that let the system evolve. STS can be effectively generated from the sos rules of the process calculus by using a unification-based approach, supporting in silico analysis of complex biological systems. Overall, this provides a formal and computational framework capable to (automatically) infer information about components that are not fully understood beforehand, and that permits the choice of the more appropriate representation language. 
To the best of our knowledge, our open-ended and inferential modeling is original in the context of bio process algebras. Indeed, in the literature, simulations and analysis have been carried out starting from completely specified models. Hence, no further information about the behaviour of the system can be inferred, in the sense we do it. A related approach, but in a different perspective, can be [7], where temporal logic is taken as a specification language and machine learning techniques are used to revise the reaction rules initially (fully) available. Another way to deal with incomplete information are discrete approximations, as claimed in [6], but in the significantly different context of the numerical approaches within formal methods. An analogous unification-based semantics construction is given in [36], in the different context of modelchecking for nominal calculi, where unknown can be the communication network.

Finally, beyond the discussed ongoing extensions, a challenging major direction to extend our approach is the use of quantitative and stochastic information as the kinetic constants of reactions and their probabilities, along the line of $[27,29]$, which would allow for more elaborated experiments.

\section{References}

1. B. Alberts, D. Bray, J. Lewis, M. Raff, K. Roberts, and J. D. Watson. Molecular Biology of the Cell. Garland, 1994.

2. P. Baldan, A. Bracciali, and R. Bruni. Bisimulation by unification. Proc. AMAST'02, LNCS 2422, 254-270, 2002.

3. P. Baldan, R. Bruni, and A. Bracciali. Symbolic equivalences for open systems. Proc. GC'04, LNCS 3267, 1-17, 2005.

4. L. Cardelli and A. Gordon. Mobile ambients. Theoretical Computer Science, 240/1:177213, 2000.

5. M. Calder, J. Hilston, and S. Gilmore. Modelling the influence of RKIP on the ERK signalling pathway using the stochastic process algebra PEPA. Trans. on Computational Systems Biology, 2006. To appear.

6. M. Calder, V. Vyshemirsky, D. Gilbert, and R. Orton. Analysis of signalling pathways using the PRISM model checker. Proc. CMSB'05, 179-190, 2005.

7. L. Calzone, N. Chabrier-Rivier, F. Fages, and S. Soliman. Machine learning biochemical networks from temporal logic properties. Trans. on Comp. Systems Biology, 2006. To appear.

8. L. Cardelli. Brane calculi-interactions of biological membranes. Proc. CMSB'04, LNCS 3082, 257-280, 2005.

9. L. Cardelli and A. Gordon. Anytime, anywhere. Modal logics for mobile ambients. Proc. POPL'00, 365-377. ACM, 2000.

10. D. Chiarugi, M. Curti, P. Degano, and R. Marangoni. VICE: A VIrtual CEll. Proc. CMSB'04, LNCS 3082, 207-220, 2004.

11. V. Danos and C. Laneve. Formal molecular biology. Th. Comp. Sci., 325(1):69-110, 2004. 
12. P. Degano, D. Prandi, C. Priami, and P. Quaglia. Beta-binders for biological quantitative experiments. Proc. QAPL'06, ENTCS. Elsevier, 2006. To appear.

13. S. Eker, M. Knapp, K. Laderoute, P. Lincoln, J. Meseguer, and K. Sonmez. Pathway Logic: Symbolic analysis of biological signaling. Proc. Pacific Symposium on Biocomputing.: 400-412, 2002

14. S. Eker, M. Knapp, K. Laderoute, P. Lincoln, and C. Talcott. Pathway Logic: Executable models of biological networks. In Proc. Workshop on Rewriting Logic and its Applications, ENCS 71, 2002.

15. F.Gadducci and U. Montanari. The Tile Model. Proof, Language and Interaction: Essays in Honour of Robin Milner, 133-166. MIT Press, 1998.

16. D. T. Gillespie. Exact stochastic simulation of coupled chemical reactions. Phys. Chem. , 81(25):2340-2361, 1977.

17. B. Gutnik, D. Pinto, and B. Ermentrout. Mathematical neuroscience: from neurons to circuits to systems. Journal of Physiology, 97:209-219, 2003.

18. O. Haller and G. Kochs. Interferon-induced Mx proteins: Dynamin- like GTPases with antiviral activity. Traffic, 3:710-717, 2002.

19. M. Hennessy and H. Lin. Symbolic bisimulations. Th. Comp. Sci., 138:353-389, 1995.

20. H. de Jong. Modeling and simulation of genetic regulatory systems: a literature review. Journal of Computational Biology, 9(1):67-103, 2002.

21. H. Kitano. Systems Biology: a brief overview. Science, 295(5560):1662-1664, 2002.

22. P. Lecca, C. Priami, P. Quaglia, B. Rossi, C. Laudanna, and G. Costantin. A stochastic process algebra approach to simulation of autoreactive lymphocyte recruitment. SIMULATION: Trans. of the society for modelling and simulation international, 80(4):273$288,2004$.

23. J. Leifer and R. Milner. Deriving bisimulation congruences for reactive systems. Proc. CONCUR'00, LNCS 1877, 243-258, 2000.

24. M. Miculan and G. Bacci. Modal logics for Brane Calculus. Proc. CMSB'06 LNCS 4210, 1-16, 2006.

25. M. Nagasaki, S. Onami, S. Miyano, and H. Kitano. Bio-calculus: its concept and molecular interaction. Genome Informatics, 10:133-143, 1999.

26. A. Phillips and L. Cardelli. A correct abstract machine for the stochastic pi-calculus. Proc. Bioconcur'04. ENTCS, 2004.

27. C. Priami. Stochastic $\pi$-calculus. The Computer Journal, 38(6): 578-589, 1995.

28. C. Priami and P. Quaglia. Beta-binders for biological interactions. Proc. CMSB'04, LNCS 3082, 21-34, 2005.

29. C. Priami and A. Regev and E. Shapiro and W. Silvermann. Application of a stochastic name-passing calculus to representation and simulation of molecular processes. Information Processing Letters, 80: 25-31, 2001.

30. A. Regev, E. Panina, W. Silverman, L. Cardelli, and E. Shapiro. Bioambients: An abstraction for biological compartements. Th. Comp. Sci., 325(1):141-167, 2004.

31. A. Regev, and E. Shapiro. Cellular Abstractions: Cells as Computation. Nature, $419,343,2002$.

32. A. Regev, W. Silverman, and E. Shapiro. Representation and simulation of biochemical processes using the pi-calculus process algebra. Proc. Pacific Symposium on Biocomputing, 459-470, 2001.

33. A. Rensink. Bisimilarity of open terms. Information and Computation, 156(1-2):345$385,2000$.

34. C. Surridge. Computational biology. Nature Insight, 420(6912):206-246, 2002.

35. C. Talcott, S. Eker, M. Knapp, P. Lincoln, and K. Laderoute Pathway logic modeling of protein functional domains in signal transduction Proc. Pacific Symposium on Biocomputing, 2004.

36. P. Yang, C. Ramakrishnan, and S. Smolka. A logical encoding of the pi-calculus: Model checking mobile processes using tabled resolution. Proc. VMCAI'03, LNCS 2575, 86-101, 2003.

37. E. L. Winnacker From Genes to Clones VCH Publishers, 1987. 


$$
\begin{aligned}
& P, Q \quad:=0|P| Q|[P]| n ! . P|n ? . P| M . P\left|\sum_{i \in I} \pi_{i} . P_{i}\right| \sum_{i \in I} M_{i} \cdot P_{i} \\
& M, N::=\operatorname{enter} n \mid \text { accept } n \mid \text { exit } n|\operatorname{expeln}| \operatorname{merge}^{+} n \mid \text { merge }^{-} n \\
& \overline{[(T+\text { enter n.P }) \mid Q] \mid\left[\left(T^{\prime}+\text { accept n.R }\right) \mid S\right] \rightarrow[[P \mid Q] \mid(R \mid S)]}(\text { enter }) \quad \overline{\left[[(T+\text { exitn.P }) \mid Q] \mid\left(T^{\prime}+\text { expel n.R }\right) \mid S\right] \rightarrow[P \mid Q] \mid[R \mid S]}(\text { exit }) \\
& \overline{\left[\left(T+\text { merge }^{+} n \cdot P\right) \mid Q\right] \mid\left[\left(T^{\prime}+\text { merge }^{-} \text {n.R }\right) \mid S\right] \rightarrow[(P \mid Q) \mid(R \mid S)]}(\text { merge }) \overline{[(T+n ! . P) \mid R]\left|\left[\left(n ? . Q+T^{\prime}\right) \mid S\right] \rightarrow[P \mid R]\right|[Q \mid S]}(\text { comm }) \\
& \frac{P|R \rightarrow Q| R}{P \rightarrow Q} \text { (par) } \quad \frac{[P] \rightarrow[Q]}{P \rightarrow Q}(\text { amb }) \quad \frac{P \rightarrow Q}{P \equiv P^{\prime}, P^{\prime} \rightarrow Q^{\prime}, Q^{\prime} \equiv Q}(\text { str })
\end{aligned}
$$

Fig. 3. A subset of syntax and semantic rules for BioAmbients

\section{A Short overview of BioAmbients}

BioAmbients [30], a variant of Mobile Ambients [4], adopts ambient constructor to model biological membranes and to identify individual components. A BioAmbients process evolves when a pair of its sub-processes interact synchronously. Interactions can be based on input and output prefixes or can involve membranes and capabilities that act on them. The last type of interactions consist of synchronizations between capabilities and the corresponding co-capabilities. Here, we restrict our interest in a subset of the calculus involving all capabilities and the sole communication between sibling ambients. Note that no name passing is allowed. The syntax and semantics rules are in Fig. 3.

The process 0 can perform no activities. The parallel composition of two processes $P \mid Q$ interleaves the execution of the activities of $P$ with those of $Q$. The two processes can also synchronize, rule (par). When the synchronization is acting on membranes, the involved capability and the corresponding co-capability must share a channel and must be in a certain structural relationship. The enter $n$ allows a membrane, that is side to side with another membrane, to enter the second one which must offer accept $n$, rule (enter). The exit $n$ allows a nested membrane to leave its containing membrane, if this one offers the corresponding expel $n$, rule (exit). The ambient offering merge ${ }^{+} n$ fuses its membrane with the membrane of another ambient offering merge $n$, rule (merge). As said before, we only allow communications between sibling ambients, i.e. the two prefixes must lay in two different membranes at the same nesting level, rule (comm). This corresponds to the sibling communication of standard BioAmbients. As usual, semantics is up to structural congruences, rule (str): + and $\|$ are monoids with 0 as neutral element, and $[0] \equiv 0, P \mid 0 \equiv P$.

\section{A.1 $\lambda$-phage life cycle}

We report three simulation traces, starting from the completely specified model of the $\lambda$-phage life cycle. relying three possible pathways, 


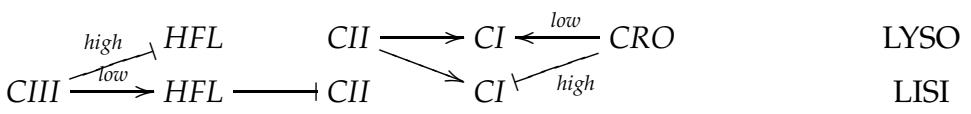

Fig. 4. Full specification of the inhibition and activation schema in Fig. 1.

according to [37]. We assume the behaviour of HFL to be as:

$$
H F L=\text { enterh_c3.0 + l_c3?.acceptc2.lysi!.0 }
$$

For readability we use the abbreviation: $D N A_{e}=$ accept dnae.0.

In Fig. 4 we complete the activation and inhibition relations of Fig. 1, as deduced by our theoretical tool: a low concentration of CIII does not inhibit the synthesis of protein HFL that, in turn, blocks the production of CII.

We show three executions leading to the lysogenic and lytic cycles. All of them are preceded by the initial phase of virus injection: the two reductions in (11) concerning the merging of the virus and the bacterium membranes.

${ }_{\lambda}\left[\right.$ VIRUS] $\left.\right|_{\text {Ecoli }}\left[\right.$ ECOLI] $\rightarrow_{\text {Ecoli }}\left[([C 3]|[C 2]|[C 1] \mid[C R O])|[D N A \lambda]|\left[D N A_{e}\right] \mid[H F L]\right]$ (11)

In case of a low concentration of CIII the simulation starts at (12):

$$
\begin{aligned}
& \text { Ecoli }\left[([\mathrm{C} 3]|[C 2]|[C 1] \mid[C R O])|[D N A \lambda]|\left[D N A_{e}\right] \mid[\mathrm{HFL}]\right](12) \\
& \rightarrow_{\text {Ecoli }}[\mathrm{CIII}[0]|[\mathrm{C} 2]|[C 1] \mid[C R O])[D N A \lambda]\left|\left[D N A_{e}\right]\right| \mathrm{hff}[\text { accept c2.lysi!.o]] (13) } \\
& \rightarrow \rightarrow_{\text {Ecoli }}\left[\left.([0]|[C 1]|[C R O])|[D N A \lambda]|\left[D N A_{e}\right]\right|_{\text {hffl }}[\mathrm{CII}[0] \mid \text { lysi!.o]] (14) }\right. \\
& \rightarrow_{\text {Ecoli }}\left[([0]|[C 1]|[C R O]) \mid{ }_{\lambda}\left[\text { exit newph.VIRUS] | expel newph] }\left|\left[D N A_{e}\right]\right|_{h f l}\left[{ }_{C I I}[0] \mid 0\right]\right]\right. \text { (15) } \\
& \rightarrow_{\text {Ecoli }}\left[\left.([0]|[C 1]|[C R O])\right|_{\lambda}[\text { VIRUS }]\left|\left[D N A_{e}\right]\right|_{h f l}\left[C_{C I I}[0] \mid 0\right]\right](16)
\end{aligned}
$$

A high concentration of $\mathrm{CIII}$ and a low of CRO leads the system to lysogeny, the simulation starts at (17):

$$
\begin{aligned}
& \text { Ecoli }\left[([\mathrm{C} 3]|[C 2]|[C 1] \mid[C R O])|[D N A \lambda]|\left[D N A_{e}\right] \mid[\text { HFL }]\right](17) \\
& \left.\rightarrow_{\text {Ecoli }}[\text { CIII }[\text { pro_c2!..0| HFL }[]]|[\mathrm{C} 2]|[C 1] \mid[C R O])|[D N A \lambda]|\left[D N A_{e}\right]\right](18) \\
& \rightarrow_{\text {Ecoli }}\left[\left(\left[\left.0\right|_{H F L}[0]\right] \mid[\text { pro_c1.0] }|[C \mathbf{C}]|[C R O])|[D N A \lambda]|\left[D N A_{e}\right]\right]\right. \text { (19) } \\
& \rightarrow_{\text {Ecoli }}\left[\left(\left[0||_{H F L}[0]\right]|[0]| \text { [high_cro?.lysi!..0 + 1_cro?.lyso!.0] | [CRO]) | [DNAג] | [DNA } A_{e}\right]\right] \text { (20) } \\
& \rightarrow \rightarrow_{\text {Ecoli }}\left[\left(\left[\left.0\right|_{H F L}[0]\right]|[0]|[\text { lyso!.0] | [0]) | [DNA } \lambda] \mid\left[D N A_{e}\right]\right](21)\right. \\
& \rightarrow_{\text {Ecoli }}\left[\left(\left[\left.0\right|_{H F L}[0]\right]|[0]|[0] \mid[0]\right) \mid\left[\text { enter dnae.0] | [DNA } \mathbf{D N}_{\mathbf{e}}\right]\right] \text { (22) }
\end{aligned}
$$

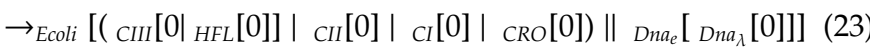

Finally, a high concentration of CRO leads the system to lysis, even with a high concentration of CIII, simulation starts at (24), continuing from (20):

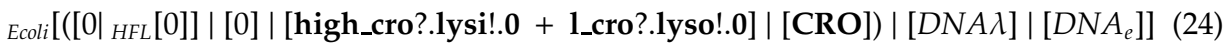

$$
\begin{aligned}
& \rightarrow \rightarrow_{\text {Ecoli }}\left[\left(\left[\left.0\right|_{H F L}[0]\right]|[0]|[\text { lysi!.0] | [0]) | [DNA } \lambda] \mid\left[D N A_{e}\right]\right](25)\right. \\
& \rightarrow_{\text {Ecoli }}\left[\left(\left[\left.0\right|_{H F L}[0]\right]|[0]|[0] \mid[0]\right) \mid\left[{ }_{\lambda}[\text { exit newph.VIRUS }] \mid \text { expel newph }\right] \mid\left[D N A_{e}\right]\right](26) \\
& \rightarrow \rightarrow_{\text {Ecoli }}\left[\left(\left[\left.0\right|_{H F L}[0]\right]|[0]|[0] \mid[0]\right) \mid{ }_{\lambda}[\text { VIRUS }] \mid\left[D N A_{e}\right]\right] \quad(27)
\end{aligned}
$$




$$
\begin{aligned}
& P, Q::=\diamond|\sigma[P]| P \circ Q|r \quad \sigma, \rho, \tau \quad::=0| a . \tau|\sigma| \tau|\ldots \quad r, s \quad::=\diamond| m \circ r \quad a \quad::=p_{n}, p_{n}^{\perp}(\sigma), e_{n}, e_{n}^{\perp}, m_{n}, m_{n}^{\perp} \ldots \\
& \overline{m_{n} . \sigma\left|\sigma_{0}[P] \circ m_{n}^{\perp} \cdot \tau\right| \tau_{0}[Q] \rightarrow \sigma\left|\sigma_{0}\right| \tau \mid \tau_{0}[P \circ Q]}(\text { mate }) \quad \overline{e_{n}^{\perp} \cdot \tau\left|\tau_{0}\left[e_{n} . \sigma \mid \sigma_{0}[P] \circ Q\right] \rightarrow P \circ \sigma\right| \sigma_{0}|\tau| \tau_{0}[Q]}(\text { exo }) \\
& \frac{P \rightarrow Q}{p_{n} \cdot \sigma\left|\sigma_{0}[P] \circ p_{n}^{\perp}(\rho) \cdot \tau\right| \tau_{0}[Q] \rightarrow \tau \mid \tau_{0}\left[\rho\left[\sigma \mid \sigma_{0}[P]\right] \circ Q\right]}(\text { phago }) \quad \frac{P \circ R \rightarrow Q \circ R}{P \circ R)} \\
& \frac{P \rightarrow Q}{r_{1} \circ r_{1}\left(r_{2}\right) \rightrightarrows s_{1}\left(s_{2}\right) . \sigma\left|\sigma_{0}\left[r_{2} \circ P\right] \rightarrow s_{1} \circ \sigma\right| \sigma_{0}\left[s_{2} \circ P\right]}(b \& r) \quad \frac{P \rightarrow \sigma[Q]}{\sigma[P] \rightarrow \sigma e m)}
\end{aligned}
$$

Fig. 5. Syntax and operational semantics of $f B C$.

\section{B Short overview of Brane Calculi}

$\mathrm{fBC}$ can be understood as an extension of BioAmbients, where membranes exhibit themselves a behaviour. Its syntax and semantics are reported in Fig. 5 and recapped below. fBC distinguishes between membrane complexes $(P, Q, \ldots)$ and membranes $(\sigma, \rho, \tau, \ldots)$. The basic complex $\sigma[P]$ consists of an active external membrane layer $\sigma$ and of complex $P$ inside the membrane. The symbol $\diamond$ denotes the null membrane complex. Complex $P \circ Q$ is obtained by composing $P$ and $Q$, while $m_{1} \circ \cdots \circ m_{k}$ is the complex consisting of a multiset of molecules $(r, s, \ldots)$. Interaction between membrane complexes happens through the active membrane layer $\sigma$. This can perform no action 0 , an action prefixed to an active layer a. $\sigma$, or like two parallel active layers $\sigma \mid \tau$ (other structure can be added). Here, we consider the actions phago ${ }_{n}\left(p_{n}\right)$ and $\operatorname{exo}_{n}\left(e_{n}\right)$, together with the corresponding coactions $p_{n}^{\perp}(\sigma)$ and $e_{n}^{\perp}$, with $n$ belonging to a set of names. Moreover, action $m_{n}$ and coaction $m_{n}^{\perp}$ modelcell merging (although it can be expressed by (phago) and (exo) -see [8]). A membrane complex $\sigma[P]$ can enter another complex $\tau[Q]$, if $\sigma$ can execute a phago $p_{n}$ action and $\tau$ the corresponding coaction $p_{n}^{\perp}(\rho)$ with the same $n$ (phago). As a result, $\sigma[P]$ is enclosed within a membrane with active part $\rho$, according to the spirit of bitonal reactions. A nested membrane complex $\tau[\sigma[P] \circ Q]$ allows the subsystem $P$ to leave the $\tau[\ldots]$ membrane complex if $\sigma$ and $\tau$ are ready to execute, respectively, $e_{n}$ and $e_{n}^{\perp}(e x o)$. Membranes and their contents merge, respectively, according to rule (mate). A membrane complex can also evolve autonomously in a system or within a membrane (rules (par) and (mem)). As standard, semantics is up to structural congruence, rule 
(str) in Tab. 3: | and $\circ$ are monoids with 0 and $\diamond$ as a neutral elements, $0[\diamond] \equiv \diamond$, see $[8]$.

\section{B.1 Virus entering the cell}

For completeness we report the fully specified model of the virus entering the cell.

$$
\begin{array}{rlrl}
\text { virus } & =p_{a} \cdot e_{a}[\text { nucap }] & \text { nucap }=\text { capsid }[v R N A] & \text { capsid }=p_{b} \mid \text { bud } \mid \text { disasm } \\
\text { cell } & =p_{a}^{\perp}\left(m_{a}\right) \mid e_{b}^{\perp}[\text { cytosol }] & \text { cytosol }=\text { endosome } \circ C C \text { endosome }=m_{a}^{\perp} \mid e_{a}^{\perp}[\diamond]
\end{array}
$$

The initial configuration of the system gives origin to the following simulation that exhibits the attended behaviour:

$$
\begin{array}{r}
p_{a} \cdot e_{a}[\text { nucap }] \circ p_{a}^{\perp}\left(m_{a}\right) \mid e_{b}^{\perp}\left[m_{a}^{\perp} \mid e_{a}^{\perp}[\diamond] \circ C C\right] \\
\rightarrow e_{b}^{\perp}\left[m_{a}\left[e_{a}[\text { nucap }]\right] \circ m_{a}^{\perp} \mid e_{a}^{\perp}[\diamond] \circ C C\right] \\
\rightarrow e_{b}^{\perp}\left[e_{a}^{\perp}\left[e_{a}[\text { nucap }]\right] \circ C C\right] \rightarrow e_{b}^{\perp}[0[\diamond] \circ \text { nucap } \circ C C]
\end{array}
$$

